\title{
MECHANICAL PRINCIPLES IN THE SURGERY OF AORTIC AND MITRAL INCOMPETENCE
}

\author{
BY \\ R. C. BROCK \\ From the Thoracic Department, Guy's Hospital
}

Received April 10, 1954

The operative treatment of valve stenosis has now been shown, as a result of large numbers of successful operations, to be very satisfactory. The operative treatment of valve incompetence is, however, quite another matter; in spite of various ingenious efforts no satisfactory method has yet been introduced. My own experience is that the mortality of these procedures is high and the results bad; from reading what has been written and from listening to the presentation of papers at society meetings I have no evidence that the results are any better in the hands of other surgeons. Indeed, at present it is difficult to feel justified in advising any patient who is suffering from predominant valve incompetence to submit to an operation for its correction. The object of this article is to record some personal experiences and to present some reasons why the methods used have failed.

Every surgeon has to decide whether he will use foreign substances, fashioned as prostheses and introduced into the body. Prostheses have been designed and used to correct valve incompetence; their presence appears to be tolerated for short periods but their later fate is quite uncertain. From the vast accumulated experience of surgeons in the fate of foreign bodies introduced into other parts of the body there is little to encourage us to hope that such substances, even if composed of a plastic, will be longer and better tolerated in the interior of the heart or of the great vessels. A long, trouble-free life would appear to be very unlikely for a young patient whose heart or aorta contains such a plastic valve.

Biologically the patient's own tissues should be much safer than a foreign material; our difficulty lies in the adaptation of tissue to serve as a useful functioning valve. The most popular substance has been pericardium, introduced into the heart or aorta in the form of a flap designed to correct the leakage of the incompetent valve. There are three reasons why this usually fails: (1) fibrosis and shrinkage; (2) unsatisfactory placing; and (3) the influence of the axial stream of regurgitant blood.

\section{(1) Fibrosis AND Shrinkage}

At first the flap is thin and supple, but after a time fibrin is deposited on it; indeed the frequent movement in the blood-stream must encourage the deposition. This fibrin later begins to organize so that the once mobile flap becomes a more solid mass of tissues with little or no pliability. When organization is complete it becomes a solid cord-like structure, rigid and shortened and not suitable for improving the function of the valve. This change was seen regularly in a series of experiments on the introduction of a pericardial flap into the aorta of dogs.

\section{(2) Unsatisfactory Placing}

This factor applies particularly to the mitral valve and has already been presented (Brock, 1952). Fig. 1 shows diagrammatically the arrangement of the mitral ring, valve cusps and orifice in relation 


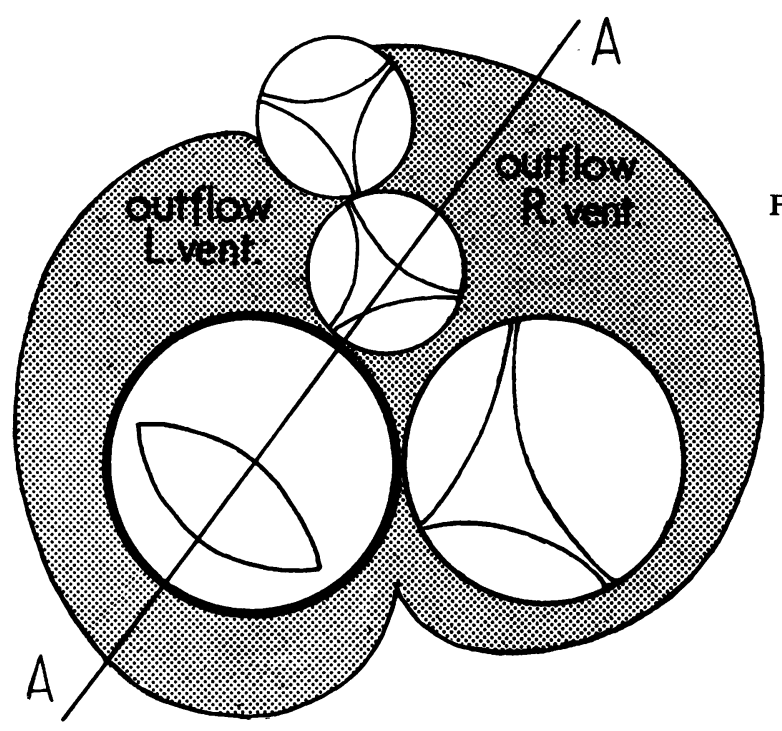

FIG. 1.-Diagram to show the basic anatomy of mitral incompetence. If a pericardial flap with two opposing sites of fixation is to be used in such a way that it is efficient, it must be at rightangles to the axis of the valve orifice; and the line $\mathbf{A}-\mathbf{A}$ indicates that inevitably, therefore, it must traverse, in front, the outflow tract of the left ventricle, the septum, and the outflow tract of the right ventricle. To avoid these structures it must be at a much less efficient angle to the optimum line A-A.

to adjacent parts of the heart. It would seem that if a pericardial flap is to be inserted through two openings so that it may form a flapper valve to close the incompetent orifice, it must be disposed at right angles to the long axis of the valve opening. It could not be parallel to this axis for the papillary muscles and their tendons are in the way. In Fig. 1 the line $\mathrm{A}-\mathrm{A}$ indicates the position that must be occupied by a pericardial flap if it is to lie transversely to the long axis of the valve orifice. It will be seen that, anteriorly, it must traverse the outflow track of the right ventricle. Clearly this is impossible.

If the septum and the right ventricle are to be avoided, the anterior fixation must be at an angle much smaller than 90 degrees to the long axis of the valve orifice and in fact forming, not the diameter of the circle of the valve ring, but a chord. Its efficiency in controlling valve incompetence diminishes as the angle diminishes. Moreover, there is no reason why such an ill-disposed flap should necessarily be flung against the imcompetent mitral orifice in systole. It may equally, or even more likely, be shot into the outflow channel of the ventricle.

In order to avoid this difficulty I made the suggestion (Brock, 1952) that as the incompetence is due fundamentally to the fact that the two cusps are too short to meet and overlap so as to give

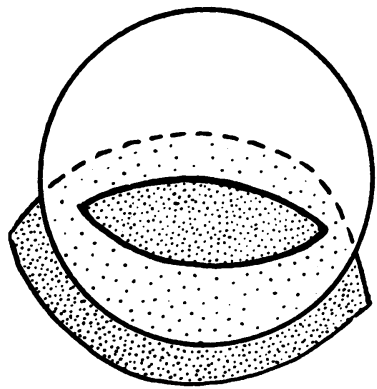

Fig. 2.-Diagram to show suggested correct mode of application of a reinforcing flap to control mitral regurgitation by extending the surface of contact of the postero-lateral cusp. mutual support, the most logical method of correcting the disability would seem to be lengthening one or both cusps so that they can reach and overlap. The antero-lateral or aortic cusp does not lend itself to such a procedure, seeing that its ventricular surface is directly related to the outflow channel. The posterior cusp, on the other hand, could either be lengthened or reinforced by a flap or some other structure introduced immediately beneath it and its function restored. Thus any material used to correct incompetence should be inserted so that it is hinged posterolaterally (Fig. 2). It was argued that a flap successfully introduced into position below the posterolateral cusp " must inevitably be flung against or across the valve orifice in systole; it cannot be diverted into the outflow channel."

My experience with such a flap has shown that although it can, technically, be introduced so as to lie in the desired position, and though it is indeed flung against or across the valve orifice in systole, this is not the whole story. In fact it is not only flung " against and 
across" the valve orifice, it may be directed into the orifice and thus cause death from acute mitral obstruction. This brings us to the third unfavourable factor.

\section{(3) The Axial Stream in Regurgitation}

The basic conception of the action of the pericardial flap during systole was that it would be acted on by a broad mass or column of blood so as to be flung firmly and securely across the incompetent orifice and thus to close it. The very force of the systolic contraction would give stability to the closure achieved by the flap. This would indeed be the case if the force of blood acted equally over the whole surface of the flap and thus applied it evenly over the incompetent mitral orifice. Actually there is a stream effect; a very powerful flow of blood pours back through the incompetent orifice and in the line of its axis; in other words an axial stream is formed which passes as a powerful jet through the valve opening. The pericardial flap is caught up in this and is projected through the opening.

This is clearly seen in the heart shown in Fig. 3, which is from a woman aged 30, who had severe mitral incompetence. The mitral orifice was found at operation to be $3 \times 1 \mathrm{~cm}$. with firm margins; a powerful stream of regurgitant blood could be felt. A large flap of pericardium was cut so as to give a valve-shaped extremity with two large pedicle-like projections. Using special needles two sutures were passed via the atrial appendage through the valve orifice and then out

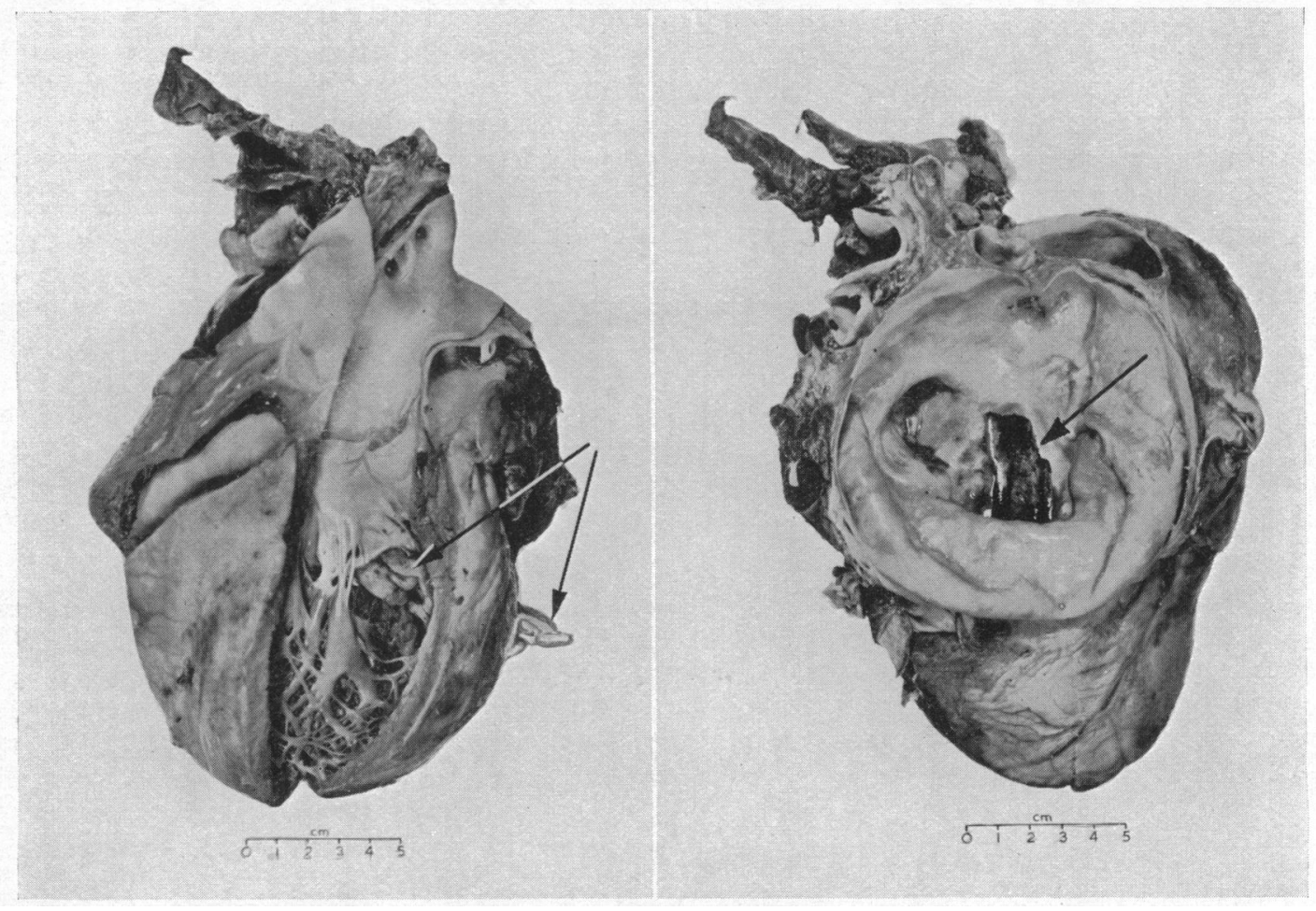

A

B

FIG. 3.-Specimen of heart in which a pericardial flap was introduced to reinforce postero-lateral cusp, as proposed in Fig. 2. In (A) the flap can be seen attached to and through the posterior wall of the ventricle and then entering the mitral orifice. (B) The valve is viewed from the atrial aspect and the flap is seen prolapsed through the mitral opening which is obstructed by it. 
through the posterior aspect of the left ventricle as high as possible. The sutures were fastened to the ends of the pericardial flap which was then drawn through the appendage, across the atrium, and through the valve orifice so as to be attached to the posterior wall of the ventricle. The two pedicles were brought out on to the posterior aspect of the ventricle and were sutured to the heart wall and to each other. In this way the cusp-like end of the flap was firmly fixed in place exactly below the posterior cusp of the mitral valve; it was certainly amply long enough to cover over the orifice in systole.

The patient made a good recovery from the operation but collapsed and died quite suddenly 24 hours later. Examination of the heart showed that the flap had been placed perfectly in position as planned and if such a flap could function efficiently this should have done so. It had, however, prolapsed through the mitral orifice and 'some $3 \mathrm{~cm}$. of it projected into the left atrium (Fig. 3). It had clearly caused acute obstruction of the mitral orifice. Its substance was good and there was very little thrombus formation.

Nature has arranged that the atrio-ventricular cusps are supported at their periphery by the papillary muscle and chordae tendineae system. Until we can reproduce such an arrangement it seems as if a pedicled flap, unsupported at the periphery, is likely to behave in the same way when exposed to the force of the axial stream. In other words some different mechanical principle will have to be employed.

\section{The Importance of Dilatation of the Valve Ring}

Irrespective of the type of valve replacement we use, it seems as if another very important mechanism acts in valve incompetence; that is dilatation of the atrio-ventricular ring. In its simplest form it is seen in the functional incompetence that occurs as a temporary state secondary to some other condition. Functional tricuspid incompetence secondary to right-sided failure from pulmonary hypertension and mitral stenosis is an excellent example. Its correction by successful medical management or its cure by operative relief of the mitral stenosis indicates its temporary, functional nature. Valve incompetence may also occur in association with myocardial weakness and dilatation in an acute febrile or toxic illness.

A striking and important observation on the significance of severe functional mitral incompetence was recently made at operation. The patient, a man aged 54, had severe calcified aortic stenosis and had been in bed for three months with right-sided heart failure that had improved considerably with treatment. Operative relief of the aortic stenosis was approached with misgiving because of the recent failure and the very large heart. When the heart was exposed the aortic stenosis was confirmed and a gradient of $60 \mathrm{~mm}$. $\mathrm{Hg}$ was demonstrated across the valve. In addition, however, a grossly powerful regurgitant stream was felt over the left atrium-by far the most severe mitral incompetence I have yet encountered at operation. The systolic pressure in the left atrium was $60 \mathrm{~mm}$. $\mathrm{Hg}$, a very high figure. The heart was very excitable and when touched with a needle the ventricles began to fibrillate; as a step in attempted resuscitation the aortic stenosis was relieved and then routine measures followed. Although sinus rhythm was restored for eleven minutes, ventricular fibrillation recurred and death ensued.

At autopsy the cusps of the mitral valve appeared quite healthy and although the ring admitted three fingers comfortably it was impossible to recognize, from the post-mortem appearances, that severe mitral incompetence had been present in life. Probably the contracture of the thick ventricular muscle in rigor mortis had caused the atrio-ventricular ring to become much smaller than in life. This illustrates the valuable extra information that can be obtained from observations on the living heart in action as opposed to those on the dead heart, which have commonly been assumed to give the truth and the whole truth. It is certain that they may fail to give accurate information of the dynamic state of the heart. In this case the aortic stenosis had been responsible for failure of the left ventricle, stretching of the atrio-ventricular ring and secondary severe functional mitral incompetence. 
When organic mitral incompetence is present, the valves do not meet because there is some absolute shortening of the cusps. As, however, the heart begins to fail the atrial ring dilates and secondary functional shortening aggravates the existing incompetence; a vicious circle is set up of worsening incompetence and increasing dilatation. It would appear, therefore, that surgical plans for the correction of mitral insufficiency must seek to correct or mitigate this passive stretching of the atrial ring.

If it were possible to reduce the size of the ring enough to bring the cusps together so that they could overlap and support each other the vicious circle might well be reversed. A functional improvement of the valve action would lead to its anatomical betterment.

\section{AORTIC INCOMPETENCE}

The action of the axial stream of regurgitant blood in mitral incompetence is seen also in aortic incompetence. In 1951 and 1952 I conducted a series of animal experiments to try and find out whether it were technically possible to introduce a pericardial flap into the aorta, whether such a flap would survive and retain its mobility, and whether it would function effectively as an accessory valve.

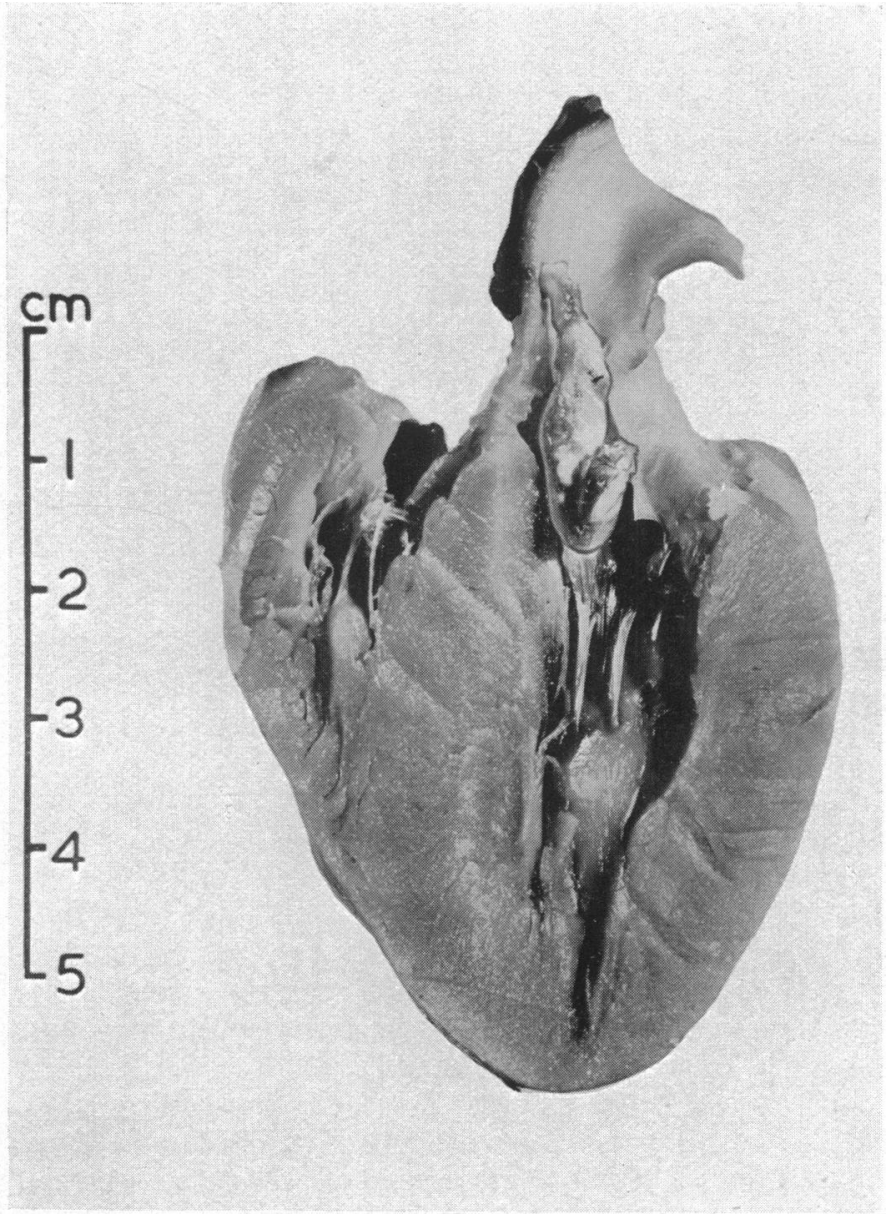

FIG. 4.-Photograph of a dog's heart in which a pedicled pericardial flap introduced into the aorta has prolapsed through the aortic orifice and caused acute left ventricular obstruction. 
It was soon found to be technically possible to introduce a pericardial flap. These flaps were used with either a single base of attachment or fixed by two pedicles on opposite sides of the aorta. In addition, it was shown that they could be well tolerated for at any rate several weeks; but as time passed the originally thin, mobile flap would become rigid and solid and would begin to organize. Ultimately it became a smaller fibrous polyp or cord.

In two dogs, however, acute and fatal left ventricular obstruction occurred from prolapse of the flap through the aortic cusps. It had been assumed that the cusps would close before the pericardial flap was flung against them in diastole. The occurrence of the fatal prolapse shows that the flap must have been drawn through the orifice just before the cusps came into firm contact. In other words an axial stream must be present for a brief time, long enough to direct the flap through the rapidly-closing orifice. Fig. 4 shows a specimen from a dog in which a single pedicled flap has prolapsed into the ventricle. This flap is so long that prolapse was favoured, but the same was observed with a much shorter bipedicled flap.

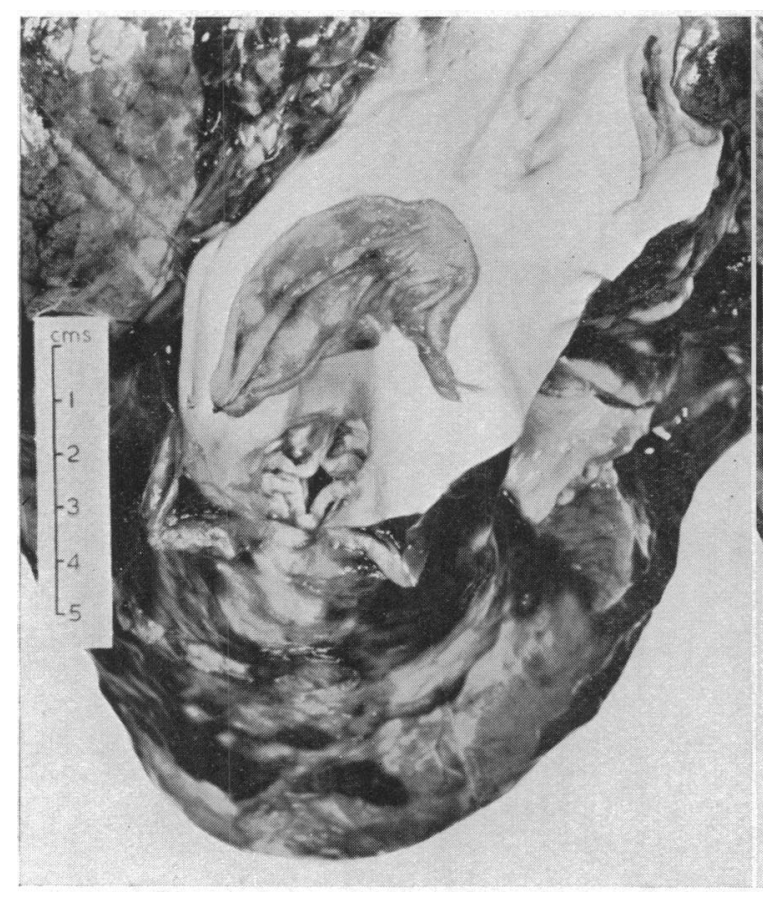

A

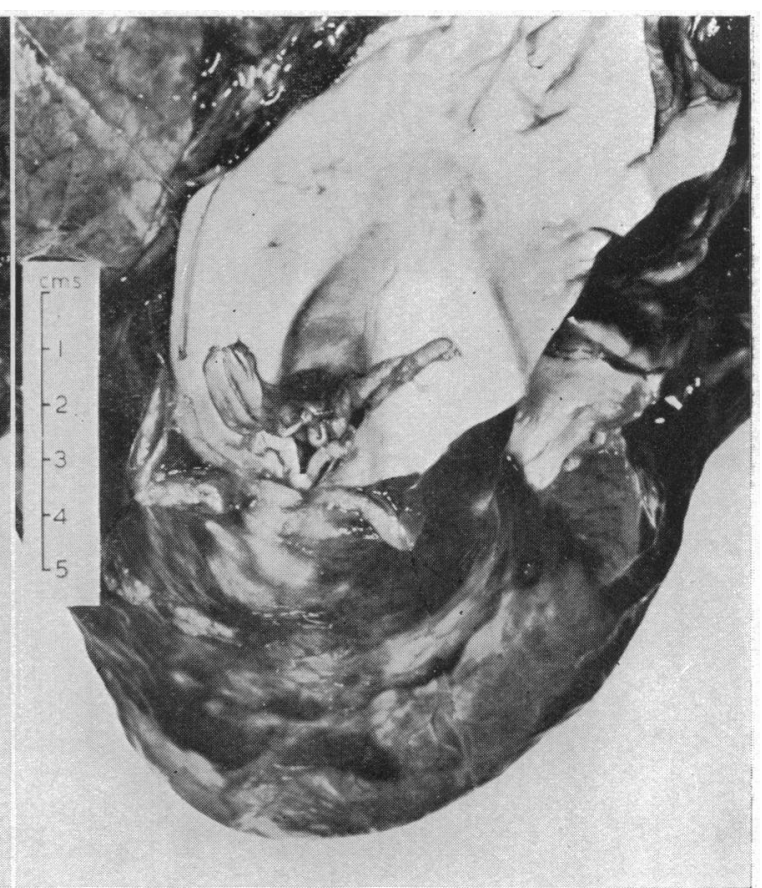

B

Fig. 5.-Photograph of a heart in which a bi-pedicled pericardial flap has been introduced into the aorta in an attempt to relieve aortic incompetence. In (A) the flap is shown flung upwards as in systole; the incompetent valve cusps are visible. (B) The flap is shown as it should be in diastole, covering the valve cusps.

The method was used on two patients and both attempts ended fatally. Fig. 5 shows the specimen from one of these and the arrangement seems very satisfactory. In (A) the incompetent valve is shown and above it, traversing the aorta and fixed at each end, is the pedicled pericardial flap turned upwards as it would be in systole. In (B) the flap is directed downwards and lies as it was hoped it would lie, covering the valve defect during diastole. Actually as soon as it was introduced into the aorta it could be felt to move up and down with a powerful " plop ", strongly suggestive of prolapsing through the valve into the ventricle and then being flung out again in systole. This performance was clearly unsatisfactory and soon the heart rapidly dilated and stopped and could not be revived. 
By the courtesy of Dr. J. K. R. McMillan of St. Thomas's Hospital the heart was examined with the apparatus he has designed and described (McMillan et al., 1952) in which it is possible to view aortic valves in action and to photograph them. The behaviour of the pericardial flap was now clearly seen; in systole it was flung up into the aorta; in diastole it was sucked down by the axial regurgitant stream through the defect and into the left ventricle. It did not spread out as a flap over the semilunar cusps, as had been visualized, but became streamlined (Fig. 6).

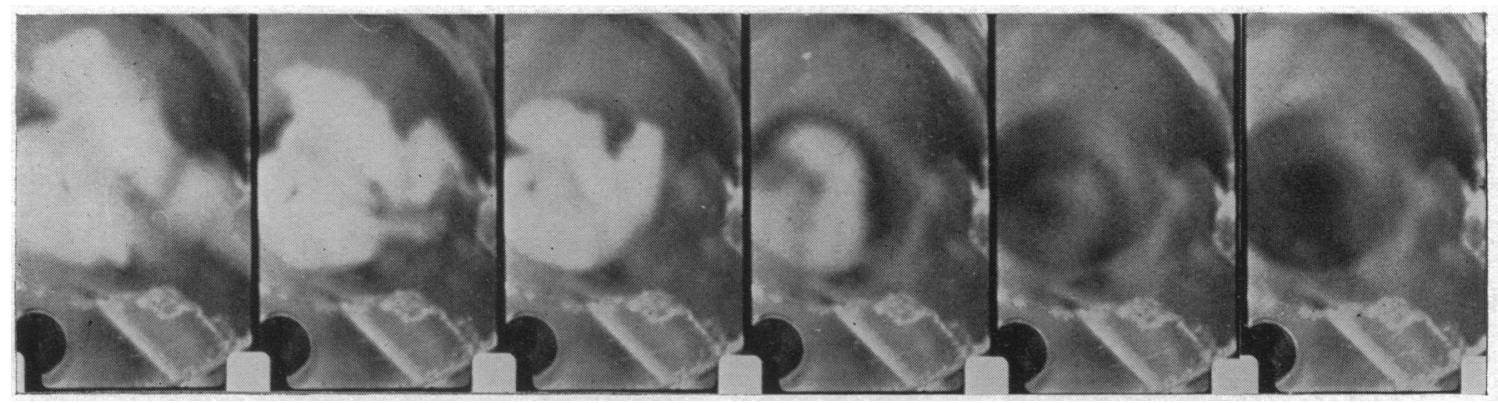

Fig. 6.-Strip from a cine-film of the pericardial flap shown in Fig. 5, in function in the special apparatus designed by Dr. McMillan. On the left the pericardial flap is seen in systole, flung up into the aorta; the pictures on the right show diastole in which the flap has actually prolapsed completely through the valve orifice into the left ventricle. Unfortunately the depth of focus of the camera does not allow the flap to be seen clearly in the pictures in diastole but the dark empty hole is shown.

Although it might be possible by careful choice of the length and placing of the flap to avoid severe prolapse, it does not seem that the flap would ever spread out so as to occlude the defect. If aortic valve incompetence is to be corrected by using autogenous or homogenous material it is clear that a different valve mechanism must be used. The ideal would be a homograft of an aortic valve. It is doubtful if this is either biologically possible or whether fibrosis and shrinkage of the valve cusps can be prevented.

The work of Lam et al. (1952) offers the possibility that, if such a valve can be inserted near the aortic outlet so that the cusps could maintain their regular function, they might survive. Herein lies sufficient promise to justify further trial and experiment. In addition it is necessary to design and test new types of valve mechanisms based on a principle different from that of the pedicled pericardial flap.

\section{REFERENCES}

Brock, R. C. (1952). Brit. Heart J., 14, 489.

Lam, C. R., Aram, H. H., and Munnell, E. R. (1952). Surg. Gynec. Obstet., 94, 129.

McMillan, J. K. R., Daley, R., and Matthews, M. B. (1952). Brit. Heart J., 14, 42. 